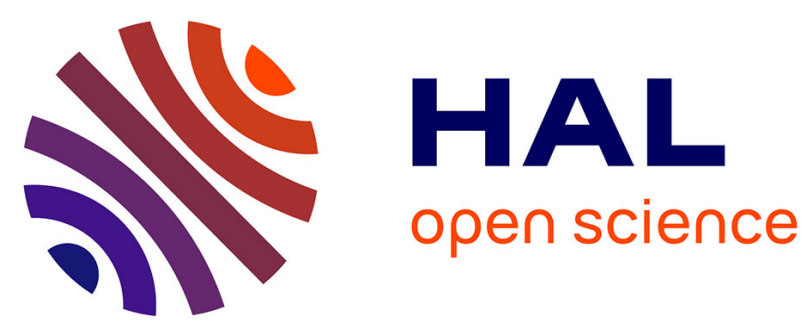

\title{
Thick fibrous composite reinforcements behave as special second-gradient materials: three-point bending of $3 \mathrm{D}$ interlocks
}

Angela Madeo, Manuel Ferretti, Francesco Dell'Isola, Philippe Boisse

\section{To cite this version:}

Angela Madeo, Manuel Ferretti, Francesco Dell'Isola, Philippe Boisse. Thick fibrous composite reinforcements behave as special second-gradient materials: three-point bending of 3D interlocks. Zeitschrift für Angewandte Mathematik und Physik, 2015, 66 (4), pp.2041-2060. 10.1007/s00033015-0496-z . hal-01144230

\author{
HAL Id: hal-01144230 \\ https://hal.science/hal-01144230
}

Submitted on 21 Apr 2015

HAL is a multi-disciplinary open access archive for the deposit and dissemination of scientific research documents, whether they are published or not. The documents may come from teaching and research institutions in France or abroad, or from public or private research centers.
L'archive ouverte pluridisciplinaire HAL, est destinée au dépôt et à la diffusion de documents scientifiques de niveau recherche, publiés ou non, émanant des établissements d'enseignement et de recherche français ou étrangers, des laboratoires publics ou privés. 


\title{
Thick fibrous composite reinforcements behave as special second-gradient materials: three-point bending of 3D interlocks
}

\author{
Angela Madeo, Manuel Ferretti, Francesco dell'Isola and Philippe Boisse
}

\begin{abstract}
In this paper, we propose to use a second gradient, 3D orthotropic model for the characterization of the mechanical behavior of thick woven composite interlocks. Such second-gradient theory is seen to directly account for the out-of-plane bending rigidity of the yarns at the mesoscopic scale which is, in turn, related to the bending stiffness of the fibers composing the yarns themselves. The yarns' bending rigidity evidently affects the macroscopic bending of the material and this fact is revealed by presenting a three-point bending test on $0^{\circ} / 90^{\circ}$ and $\pm 45^{\circ}$ specimens of composite interlocks. These specimens differ one from the other for the different relative direction of the yarns with respect to the edges of the sample itself. Both types of specimens are independently seen to take advantage of a second-gradient modeling for the correct description of their macroscopic bending modes. The results presented in this paper are essential for the setting up of a correct continuum framework suitable for the mechanical characterization of composite interlocks. The few second-gradient parameters introduced by the present model are all seen to be associated with peculiar deformation modes of the mesostructure (bending of the yarns) and are determined by inverse approach. Although the presented results undoubtedly represent an important step toward the complete characterization of the mechanical behavior of fibrous composite reinforcements, more complex hyperelastic second-gradient constitutive laws must be conceived in order to account for the description of all possible mesostructure-induced deformation patterns.
\end{abstract}

Keywords. Fibrous composite interlocks · Second-gradient models · Three-point bending test · Yarns' mesoscopic bending stiffness.

\section{Introduction}

Generalized continuum models are known to be able to account for some effects of microstructure on the overall mechanical behavior of heterogeneous materials since the pioneering works of Gabrio Piola [1]. He was indeed led, by deep physical arguments, ${ }^{1}$ to consider gradients of displacement higher than the first as needed independent variables in the constitutive equations for continuous media. Later on, second-gradient models continued to be used for describing the behavior of materials whose microstructure has a non-negligible influence on macroscopic phenomena, as shown by Mindlin and Toupin in [3-6]. At the same time, other generalized continuum theories were introduced which accounted for more general kinematical frameworks with respect to Cauchy and second-gradient theories. Such theories were known as micromorphic theories (see, e.g., $[7,8]$ ) and explicitly accounted for the possibility of describing deformations associated with the microstructure of the considered continua by supplementing the

The authors thank INSA-Lyon for the financial support assigned to the project BQR 2013-0054 "Matériaux Méso et Micro-Hétérogènes: Optimisation par Modèles de Second Gradient et Applications en Ingénierie". The second author thanks the Rhône-Alpes Région for partial funding of this project. The first and the last authors thank CRNS-INSIS for support to the PEPS project "Modélisation en Second Gradient des Renforts Fibreux de Composites".

${ }^{1}$ Actually, he considered a kind of non-local theory which has been later called peridynamics (see [2]). 
classical displacement field with additional higher-order tensors permitting the description of micromotions. Micromorphic models are more general than second-gradient ones in the sense that the microscopic motions are completely independent of the macroscopic ones due to the introduced extended kinematics. It is also known that second-gradient models can be obtained, in a sense, as "limits" of micromorphic models by imposing suitable kinematical constraints relating the micromorphic tensor to the gradient of displacement (see, e.g., [9-14]).

After the flourishing activity stemming from the original ideas of Piola [1] and associated with the works of Mindlin [3,4,7], Toupin [5,6], Cosserat brothers [15] and Germain [16,17], the research flow associated with the development and exploitation of generalized continuum theories stopped for some decades. In the last years, such theories came back onto the scene with renewed force and are presently one of the most promising tools for engineering applications concerning the characterization of microstructured materials (see, e.g., [11,18-29]). Indeed, some newly designed materials and structures try to exploit the micro-macro coupling to get peculiar and exotic macroscopic behaviors: The obtained materials are sometimes called metamaterials. The new concept of metamaterials is attracting more and more the interest of physicists and mechanicians (see, e.g., [30,31]). Some of these materials are obtained by suitably assembling multiple individual simple elements, but arranged in complex (quasi-)periodic substructures in order to show specifically designed mechanical properties. Indeed, the particular shape, geometry, size, orientation and arrangement of their constituting elements can affect, e.g., the propagation of waves of light or sound in a not-already-observed manner, creating material properties which cannot be found in conventional materials. Particularly promising in the design of metamaterials are those microstructures which present high contrast in microscopic properties: These microstructures, once homogenized, may produce generalized continuum models (see, e.g., [19-22,32,33]). The microstructures of such metamaterials, although remaining quasi-periodical, are conceived so that some of the physical microproperties characterizing their behavior diverge when the size of the REV tends to zero, while simultaneously some other properties are vanishing in the same limit.

One of the most promising fields of application of generalized continuum theories is that of the study of the mechanical behavior of fibrous composite reinforcements. Such materials are indeed constituted by two orders of yarns which have very high elongation stiffness, but very low shear stiffness: Because of their sometimes exotic behavior, they can be regarded as a kind of metamaterial. The strong contrast which they show in their mechanical properties at the mesostructure level is such that the homogenized continuum model must necessarily be included at least in the framework of second-gradient theories. Indeed, it is now generally accepted (see [18]) that classical Cauchy theories are not sufficient for the description of specific deformation patterns usually observed in fibrous composites. In particular, we refer to those concentration of strains in thin transition layers which are observed in bias extension test and which can be related to flexural strains of the yarns and so, indirectly, to the flexural strain of the thin fibers constituting the yarns themselves. It must be specified that we are interested in this paper to the mechanical characterization of fibrous composite reinforcements. This means that only the fibrous material with no organic resin is considered here. Indeed, fibrous composites can be easily shaped to conceive complex engineering pieces and such a shape is then maintained by injection and curing of a thermoset resin. The behavior of the final material (fibrous reinforcement + matrix) is quite different from that of the raw fibrous composite as discussed, e.g., in [34].

In [35], some experimental evidences about 3D fibrous composite reinforcements are described and it is seen how a first-gradient Cauchy model is not able to account for all the observed phenomenological features. In the same paper, it is also suggested that flexural deformation of the yarns may be involved in the observed phenomenology which is not encompassed by Cauchy models. Moreover, in [36], it is proposed a continuum model where flexural deformation of yarns is accounted for by means of deformation energies depending on the second gradient of displacement. It is worth noticing that the model proposed in [36] is coherent with what has been done in [37] for only one family of fibers. Therefore, the heuristic treatment proposed in [35] (which included in the 3D model some beam elements in order to partially account for 
experimental evidence) seems well grounded as it suggests a 3D second-gradient continuum model which coherently explains phenomenology.

In this paper, we:

- formulate a first 3D extension of the 2D model presented in [36] by introducing suitable quadratic second-gradient deformation energy dependences,

- adopt the same deformation energy introduced by [35] for the description of the first-gradient, hyperelastic, orthotropic constitutive behavior,

- introduce a 3D generalization of the numerical integration scheme proposed in [18] to describe the three-point bending test with fixed cylindrical supports for which [35] supplies experimental evidence and first-gradient numerical simulations,

- calibrate second-gradient constitutive parameters to describe at best the experimental mechanical behavior of the three-point bending of $0^{\circ} / 90^{\circ}$ and $\pm 45^{\circ}$ fibrous composite interlocks.

The performed numerical simulations were obtained by interfacing the solid mechanics module (used to model contact and first-gradient constitutive behavior) with the weak form PDE module (used to implement second-gradient constitutive laws) in $\mathrm{COMSOL}^{\circledR}$. Second-gradient effects were obtained by using suitable Lagrange multipliers linking the introduced micromorphic kinematical descriptors to suitable orthotropic invariants of the right Cauchy-Green deformation tensor.

Constrained micromorphic models regularize numerical integration schemes which become more stable; therefore, they are a powerful method for formulating efficient simulation tools. Moreover, constrained micromorphic models convey into the modeling procedure a physically important information about the microstructure of considered mechanical systems. The macroscopic effects of the microstructure are particularly energetically relevantly close to the contact regions of the specimen with the rigid supports. Indeed, in such zones, transition layers are visible in the second-gradient solutions which allow for a smooth transition of the out-of-plane shear angle from one value to the other. Sharp variations of out-ofplane shear strain are instead observed in the first-gradient solution as they are energetically inexpensive. Therefore, a spatially concentrated coupling between microstructure and macro-displacement in the particular mechanical and geometrical configurations considered results in a macroscopical difference between first- and second-gradient mechanical behavior.

It must be remarked that the presence of microstructure may produce other relevant phenomena which are not included in the generalized continuum model presented in the present paper. More particularly, the presence of microstructure could induce loss of stability, buckling and post-buckling phenomena which may occur in considered structures: While refraining here to attempt to model such phenomena, we want to mention that by using methods similar to those presented in [38-40], also this modeling challenge may be confronted. Moreover, we also remark that the proposed second-gradient model is intrinsically macroscopic and that some micro-macro identifications should be envisaged following methods of the type presented, e.g., in [19,22,32,41-49].

In conclusion, we can state that the numerical difficulties found when applying Cauchy models are a symptom of their weakness in the modeling capabilities of complex physical phenomena. Introducing second-gradient models, one simultaneously obtains a twofold effect (i) to enlarge the scope of applicability of continuum theories and (ii) to improve the efficiency of introduced numerical integration schemes.

\section{Kinematics}

In this section, we are interested in the introduction of the correct kinematical framework which is needed to describe the deformation of three-dimensional interlocks. To do so, we follow the reasonings proposed in [36] for the case of two-dimensional networks in which suitable second-gradient energies are proposed which account for the effect of yarns' bending stiffness on the deformation of the considered $2 \mathrm{D}$ woven fabrics. 


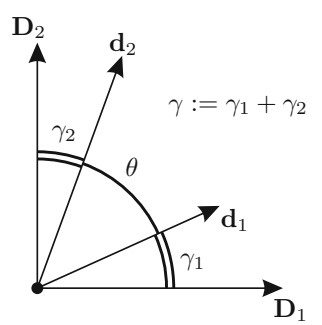

FIG. 1. Eulerian yarn vectors $\mathbf{d}_{1}$ and $\mathbf{d}_{2}$ : The angle $\theta$ is the angle between yarns in the current configuration, $\gamma=\gamma_{1}+\gamma_{2}$ is the total angle variation with respect to the reference configuration

Fibrous composite interlocks are constituted by different layers of thin woven fabrics which are held together by a complex weaving pattern. To account for the fact that such materials show two privileged material directions, we introduce two orthonormal vectors $\mathbf{D}_{1}$ and $\mathbf{D}_{2}$ which represent the warp and weft directions of the yarns constituting the $2 \mathrm{D}$ woven fabrics in the reference configuration. These weaving directions are assumed to be the same for all points in the considered woven specimen. A third direction can be introduced as $\mathbf{D}_{3}=\mathbf{D}_{1} \times \mathbf{D}_{2}$ : It is worth noticing that while $\mathbf{D}_{1}$ and $\mathbf{D}_{2}$ actually identify the pattern of the yarns in the undeformed configuration, the third unit vector $\mathbf{D}_{3}$ does not necessarily represent a material direction. The quoted set of unit normal vectors is known to be worth to describe the reference configuration of an orthotropic material (see, e.g., [50]). Once the Lagrangian unit vectors are introduced, we can define the corresponding Eulerian vectors as:

$$
\mathbf{d}_{1}=\mathbf{F} \cdot \mathbf{D}_{1}, \quad \mathbf{d}_{2}=\mathbf{F} \cdot \mathbf{D}_{2}, \quad \mathbf{d}_{3}=\mathbf{F} \cdot \mathbf{D}_{3},
$$

where $\mathbf{F}=\nabla \boldsymbol{\chi}$ is the gradient of the usual placement map $\chi$. The vectors $\mathbf{d}_{1}, \mathbf{d}_{2}$ and $\mathbf{d}_{3}$ are the pushforward in the current configuration of the vectors $\mathbf{D}_{1}, \mathbf{D}_{2}$ and $\mathbf{D}_{3}$, respectively. It is worth to stress the fact that while the vectors $\mathbf{d}_{1}$ and $\mathbf{d}_{2}$ represent the current directions of the warp and weft, the vector $\mathbf{d}_{3}$ cannot be related to privileged directions inside the considered orthotropic material.

We can summarize by saying that the kinematics of the considered continuum is univocally determined by the introduction of a suitably regular placement field $\chi: B_{0} \rightarrow \mathbb{R}^{3}$ which maps the Lagrangian configuration $B_{0} \subset \mathbb{R}^{3}$ of the considered body into the 3D Euclidean space. The deformation of the body is hence completely described by means of the deformation gradient $\mathbf{F}=\nabla \boldsymbol{\chi}$ as in classical continuum mechanics. In this framework, if one introduces an orthonormal basis $\left\{\mathbf{D}_{1}, \mathbf{D}_{2}, \mathbf{D}_{3}\right\}$, the corresponding deformed vectors $\left\{\mathbf{d}_{1}, \mathbf{d}_{2}, \mathbf{d}_{3}\right\}$ are immediately found by means of Eq. (1). The fact of identifying two of the Lagrangian material vectors (namely $\mathbf{D}_{1}$ and $\mathbf{D}_{2}$ ) with the reference directions of yarns will be seen to be useful to describe in an intuitive way the deformation of the considered orthotropic material.

In fact, with reference to Fig. 1 for the definition of the angles $\theta$ and $\gamma$, it is possible to remark that the shear strain $S$ can be related to the total angle variation $\gamma$ according to the formula

$$
S=\mathbf{d}_{1} \cdot \mathbf{d}_{2}=\left|\mathbf{d}_{1}\right|\left|\mathbf{d}_{2}\right| \cos (\theta)=\left|\mathbf{d}_{1}\right|\left|\mathbf{d}_{2}\right| \sin (\gamma),
$$

where $\gamma=\gamma_{1}+\gamma_{2}$ is the total angle variation field between the two orders of yarns from the reference configuration to the current one and $|\cdot|$ represents the length of considered vectors. Analogously, $\lambda_{1}=\left|\mathbf{d}_{1}\right|$ and $\lambda_{2}=\left|\mathbf{d}_{2}\right|$ are a measure of the yarns' stretches: Indeed, the elongations of the two orders of yarns with respect to the reference configuration can be easily obtained as $\lambda_{1}-1$ and $\lambda_{2}-1$, respectively.

\section{Second-gradient energy density for $3 \mathrm{D}$ interlocks}

The aim of this section is to introduce constitutive laws which are suitable to describe at best the mechanical behavior of $3 \mathrm{D}$ fibrous composite reinforcements. It will be shown that a second-gradient 
constitutive law which is able to account for the in-plane and out-of-plane bending stiffnesses of the yarns is indeed necessary to correctly model the mechanical behavior of such materials. Following what was done in [36] for the $2 \mathrm{D}$ case, we suppose that the deformation energy density $W$ depends on the deformation tensor and on its gradient by means of the following additive decomposition:

$$
W(\mathbf{F}, \nabla \mathbf{F})=W_{\mathrm{I}}(\mathbf{F})+W_{\text {II }}(\mathbf{F}, \nabla \mathbf{F}),
$$

where $W_{\mathrm{I}}$ and $W_{\mathrm{II}}$ are the first- and second-gradient energies, respectively.

In order to determine a suitable constitutive expression for the first-gradient energy $W_{\mathrm{I}}$, we start recalling the representation theorem for orthotropic materials (see [50]) which states that the first-gradient energy for an orthotropic material can take the following functional form:

$$
W_{\mathrm{I}}(\mathbf{F})=W_{\mathrm{I}}\left(i_{1}, i_{4}, i_{6}, i_{8}, i_{9}, i_{10}\right),
$$

where

$$
\begin{aligned}
& i_{4}=\mathbf{D}_{1} \cdot \mathbf{C} \cdot \mathbf{D}_{1}=\lambda_{1}^{2}, \quad i_{6}=\mathbf{D}_{2} \cdot \mathbf{C} \cdot \mathbf{D}_{2}=\lambda_{2}^{2}, \quad i_{8}=\mathbf{D}_{1} \cdot \mathbf{C} \cdot \mathbf{D}_{2}=S, \\
& i_{9}=\mathbf{D}_{1} \cdot \mathbf{C} \cdot \mathbf{D}_{3}, \quad i_{10}=\mathbf{D}_{2} \cdot \mathbf{C} \cdot \mathbf{D}_{3}, \quad i_{1}=\operatorname{tr}(\mathbf{C}),
\end{aligned}
$$

are the invariants of the right Cauchy-Green deformation tensor for an orthotropic material and where $\mathbf{C}=\mathbf{F}^{T} \cdot \mathbf{F}$ is the classical right Cauchy-Green deformation tensor. It is worth noticing that the first three invariants, respectively, coincide with the square of the stretches in the yarns' direction and with the shear strain. The invariants $i_{9}$ and $i_{10}$, on the other hand, are related to the out-of-plane angle variations of the two orders of yarns, and their spacial gradient can be related to the out-of-plane bending of the yarns. Specific constitutive laws for the first-gradient energy which fit available experimental data will be given in the next subsection.

As far as the second-gradient energy is concerned, a general class of expressions which can be considered is of the type

$$
W_{\text {II }}(\mathbf{F}, \nabla \mathbf{F})=W_{\text {II }}\left(\nabla i_{1}, \nabla i_{4}, \nabla i_{6}, \nabla i_{8}, \nabla i_{9}, \nabla i_{10}\right) .
$$

In the next subsection, we will point out some reasonings which will allow us to consider simpler constitutive expressions for the second-gradient energy which are suitable to describe the overall behavior of the considered interlock subjected to three-point bending.

\subsection{Constitutive choice for the first-gradient energy}

Following what was done in [35], we introduce some specific functions of the introduced invariants which are relatively simple to be determined by means of suitable experimental settings:

$$
\begin{aligned}
I_{\text {elong }}^{1} & =\ln \left(\sqrt{i_{4}}\right), \quad I_{\text {elong }}^{2}=\ln \left(\sqrt{i_{6}}\right), & I_{\mathrm{sh}}^{p} & =\frac{i_{8}}{\sqrt{i_{4} i_{6}}}, \\
I_{\mathrm{sh}}^{t 1} & =\frac{i_{9}}{\sqrt{i_{4} i_{11}}}, & I_{\mathrm{sh}}^{t 2} & =\frac{i_{10}}{\sqrt{i_{6} i_{11}}}, \quad I_{\mathrm{comp}}=\ln \left(\sqrt{\frac{i_{3}}{i_{4} i_{6}}}\right),
\end{aligned}
$$

where the invariants which have not been previously introduced are defined as

$$
i_{3}=\operatorname{det}(\mathbf{C}), \quad i_{11}=\mathbf{D}_{3} \cdot \mathbf{C} \cdot \mathbf{D}_{3} .
$$

Indeed, considering an energy which depends on the quantities appearing in (5) is equivalent to consider a functional dependence of the type (3). In fact, as shown in [50], the additional two invariants defined in (6) depend on the previously introduced ones by means of the following relationships

$$
i_{11}=i_{1}-i_{4}-i_{6}, \quad i_{3}=\left(i_{4} i_{6}-i_{8}^{2}\right)\left(i_{1}-i_{4}-i_{6}\right)+2 i_{8} i_{9} i_{10}-i_{6} i_{9}^{2}-i_{4} i_{10}^{2} .
$$

The interest of introducing a particular functional dependence of the strain energy density on the invariants (4) through the introduction of the quantities (5) can be found in the fact that these quantities 
can be easily measured by means of suitable experimental setups. The two quantities $I_{\text {elong }}^{1}$ and $I_{\text {elong }}^{2}$ are directly related to yarns elongations $\lambda_{1}=\sqrt{i_{4}}$ and $\lambda_{2}=\sqrt{i_{6}}$. As for the second quantity, it can be checked that $I_{s h}^{p}=\sin (\gamma)$ [see also Eq. (2)]: This means that it can be directly related to the shear angle variation between yarns. Analogously, $I_{\mathrm{sh}}^{t 1}$ and $I_{\mathrm{sh}}^{t 2}$ represent the out-of-plane angle variations of the two orders of yarns and are thus related to out-of-plane shear modes. Finally, $I_{\text {comp }}$ represents a normalized volume variation which can be directly related to a compression deformation mode. The possibility of performing simple elementary measurements on the quantities (5) allows the conception of constitutive laws which characterize the behavior of composite interlocks and which show considerable agreement with the available experimental evidences. In [35], it is proposed a constitutive expression of the first-gradient deformation energy of the type

$$
W_{\mathrm{I}}=W_{\text {elong }}^{1}+W_{\text {elong }}^{2}+W_{\text {comp }}+W_{\mathrm{sh}}^{p}+W_{\mathrm{sh}}^{t 1}+W_{\mathrm{sh}}^{t 2}
$$

where

$$
\begin{aligned}
& W_{\text {elong }}^{1}= \begin{cases}\frac{1}{2} K_{\text {elong }}^{0}\left(I_{\text {elong }}^{1}\right)^{2} & \text { if } I_{\text {elong }}^{1} \leq I_{\text {elong }}^{0} \\
\frac{1}{2} K_{\text {elong }}\left(I_{\text {elong }}^{1}-I_{\text {elong }}^{0}\right)^{2}+\frac{1}{2} K_{\text {elong }}^{0} I_{\text {elong }}^{\mathrm{I}} I_{\text {elong }}^{0} & \text { if } I_{\text {elong }}^{1}>I_{\text {elong }}^{0},\end{cases} \\
& W_{\text {elong }}^{2}= \begin{cases}\frac{1}{2} K_{\text {elong }}\left(I_{\text {elong }}^{2}\right)^{2} & \text { if } I_{\text {elong }}^{2} \leq I_{\text {elong }}^{0} \\
\frac{1}{2} K_{\text {elong }}^{1}\left(I_{\text {elong }}^{2}-I_{\text {elong }}^{0}\right)^{2}+\frac{1}{2} K_{\text {elong }}^{0} I_{\text {elong }}^{\mathrm{II}} I_{\text {elong }}^{0} & \text { if } I_{\text {elong }}^{2}>I_{\text {elong }}^{0},\end{cases} \\
& W_{\mathrm{comp}}=K_{\mathrm{comp}}\left(\left(1-\frac{I_{\mathrm{comp}}}{I_{\mathrm{comp}}^{0}}\right)^{-q}-q \frac{I_{\mathrm{comp}}}{I_{\mathrm{comp}}^{0}}-1\right) \\
& W_{\mathrm{sh}}^{p}= \begin{cases}K_{\mathrm{shp}}^{12}\left(I_{\mathrm{sh}}^{p}\right)^{2} & \text { if }\left|I_{\mathrm{sh}}^{p}\right| \leq I_{\mathrm{sh}}^{\mathrm{p} 0} \\
K_{\mathrm{shp}}^{21}\left(1-\left|I_{\mathrm{sh}}^{p}\right|\right)^{-p}+W_{\mathrm{shp}}^{0} & \text { if }\left|I_{\mathrm{sh}}^{p}\right|>I_{\mathrm{sh}}^{p 0}\end{cases} \\
& W_{\text {sh }}^{t 1}= \begin{cases}\frac{1}{2} K_{\text {sht1 }}^{12}\left(I_{\text {sh }}^{t 1}\right)^{2} & \text { if }\left|I_{\text {sh }}^{t 1}\right| \leq I_{\text {sht1 }}^{0} \\
K_{\text {sht1 }}^{22}\left(I_{\text {sh }}^{t 1}\right)^{2}+K_{\text {sht1 }}^{21}\left|I_{\text {sh }}^{t 1}\right|+W_{\text {sht1 }}^{0} & \text { if }\left|I_{\text {sh }}^{t 1}\right|>I_{\text {sht1 }}^{0}\end{cases} \\
& W_{\mathrm{sh}}^{t 2}= \begin{cases}\frac{1}{2} K_{\mathrm{sht} 2}^{12}\left(I_{\mathrm{sh}}^{t 2}\right)^{2} & \text { if }\left|I_{\mathrm{sh}}^{t 2}\right| \leq I_{\mathrm{sht} 2}^{0} \\
K_{\mathrm{sht} 2}^{22}\left(I_{\mathrm{sh}}^{t 2}\right)^{2}+K_{\mathrm{sht} 2}^{21}\left|I_{\mathrm{sh}}^{t 2}\right|+W_{\mathrm{sht} 1}^{0} & \text { if }\left|I_{\mathrm{sh}}^{t 2}\right|>I_{\mathrm{sht} 2}^{0}\end{cases}
\end{aligned}
$$

In the previous formulas, all the quantities which have not been introduced before are constant. It is worth noticing that the elongation energies $W_{\text {elong }}^{1}$ and $W_{\text {elong }}^{2}$ are defined in such a way that a threshold value $I_{\text {elong }}^{0}$ exists for which the yarns' rigidity is smaller for small elongations than for higher ones $\left(K_{\text {elong }}^{0}<K_{\text {elong }}\right.$ ). This constitutive choice allows to take into account the fact that the yarns are not initially straight due to weaving and they can hence initially be elongated more easily. The elongation threshold $I_{\text {elong }}^{0}$ corresponds to the configuration in which the yarns are completely straightened and start showing a higher resistance to deformation. The need of introducing such elongation strain energy densities is related to the fact that they actually carefully describe the response of the woven yarns to elongation. Nevertheless, the elongation of yarns is a mechanism which is definitely less important than the deformation mechanism associated with the angle variations between the two orders of yarns. We can actually say that, in most of the experimental tests, the considered yarns can be considered 
almost inextensible with respect to the observed predominant shear strains. As it will be better pointed out in the next subsection, this feature, which is peculiar of fibrous composite reinforcements, will be essential for choosing a simplified constitutive expression for the second-gradient energy. We remark that the first-gradient energy given in Eq. 7 is non-rank one-convex: The fact of considering an additional second-gradient energy hence provides a regularization of the considered problem (see, e.g., [51-53]) which becomes well posed.

\subsection{Constitutive choice for the second-gradient energy}

In this subsection, we specify the constitutive expression which we will use to model the mechanical behavior of 3D composite interlocks. To do so, we start considering some results recently proposed in [36] for $2 \mathrm{D}$ woven composites. In the quoted paper, it is shown that a suitable 2D second-gradient energy which is able to account for in-plane bending stiffness of the yarns at the mesoscopic scale is of the type

$$
W_{\text {II }}(\mathbf{F}, \nabla \mathbf{F})=\frac{1}{2} A_{\lambda}\left(\left|\nabla \lambda_{1}\right|^{2}+\left|\nabla \lambda_{2}\right|^{2}\right)+\frac{1}{2} A_{S}|\nabla S|^{2},
$$

where $A_{\lambda}$ and $A_{S}$ are positive constants. This energy has been shown to be a good choice for the description of the mechanical behavior of $2 \mathrm{D}$ woven composites due to its convexity with respect to $\nabla \mathbf{F}$ which guarantees well posedness of the resulting differential problem. A second-gradient energy of this type has also been used in [18] to model the bias extension test on $2 \mathrm{D}$ woven composites. It is clear that when considering inextensible yarns, the gradient of elongations are vanishing and the second-gradient strain energy density thus reduces to

$$
W_{\text {II }}(\mathbf{F}, \nabla \mathbf{F})=\frac{1}{2} A_{S}|\nabla S|^{2}=\frac{1}{2} A_{S}\left|\nabla i_{8}\right|^{2} .
$$

In [36], it is also shown that in the limit case of inextensible yarns, an alternative to the strain energy density (9) is given by

$$
W_{\mathrm{II}}(\mathbf{F}, \nabla \mathbf{F})=\frac{1}{2} A_{g}\left(\left|\mathbf{g}_{1}\right|^{2}+\left|\mathbf{g}_{2}\right|^{2}\right)
$$

where

$$
\mathbf{g}_{1}=\kappa_{1} \boldsymbol{\nu}_{1}, \quad \mathbf{g}_{2}=\kappa_{2} \boldsymbol{\nu}_{2}
$$

are two vectors which account for the bending of the yarns at the mesoscopic level, and $A_{g}$ is a positive constant. In the last formulas $\kappa_{1}$ and $\kappa_{2}$ are the in-plane bending strains of the two orders of yarns and $\boldsymbol{\nu}_{1}$ and $\boldsymbol{\nu}_{2}$ are vectors orthogonal to the current yarn directions $\mathbf{d}_{1}$ and $\mathbf{d}_{2}$, respectively, (see [36] for more details). Direct comparison of equations (9) and (10) allows to conclude that in the case of almost inextensible yarns, the fact of considering an energy accounting for the gradient of the shear angle variation is equivalent to consider an energy accounting for the bending of the two orders of yarns at the mesoscopic level. This interpretation is intriguing since it provides a clear interpretation of the deformation mechanisms which take place at the mesoscopic level when considering woven fabrics.

By extension of the previous reasoning, we consider the following expression for the second-gradient energy to be used for accounting for out-of-plane bending stiffness of the yarns in 3D composite interlocks

$$
W_{\mathrm{II}}(\mathbf{F}, \nabla \mathbf{F})=\frac{1}{2} A_{S}^{t 1}\left|\nabla i_{9}\right|^{2}+\frac{1}{2} A_{S}^{t 2}\left|\nabla i_{10}\right|^{2} .
$$

By this constitutive choice, we are considering that the tows are almost inextensible (negligible elongations compared to the shear strains) and that the predominant second-gradient deformation modes are the outof-plane bending of the yarns at the mesoscopic level. This is sensible if one considers that the traction stiffness of carbon fibers is much higher than the shear resistance that yarns experience while varying the angle between them. 
As a matter of fact, the constitutive choice (11) for the second-gradient strain energy density deserves more accurate investigations in future works in order to be generalized to describe any observable material behavior of thick composite interlocks. Actually, even if the predominant mesostructure-related deformation mechanisms which are activated in the three-point bending test are the out-of-plane bending of the yarns, it is possible that other second-gradient mechanisms could be activated when considering other loading and/or boundary conditions. In order to explore all these possibilities, other independent macroscopic tests need to be conceived which are able to unveil such supplementary material behaviors taking place at the mesoscopic level. A fully realistic constitutive choice for the generalized elasticity parameters remains a big challenge for mechanicians and it constitutes an open field of research. Despite the simplicity of the constitutive choice made here, nonlinear material behaviors are likely to occur also for second-gradient deformation mechanisms. If Eq. (11) is well adapted for describing the macroscopic effect of the mesostructure when considering a macroscopic bending of the specimen, it is possible that more general expressions (including a dependence of the elastic second-gradient parameters on the first-gradient strain and/or more complicated functional expressions for the strain energy density) will be needed to describe the behavior of interlocks when subjected to arbitrary loading and boundary conditions.

\section{Least action principle and principle of virtual powers}

Once the kinematics and the adopted constitutive laws for 3D orthotropic materials have been introduced, we can introduce the action functional as

$$
\mathcal{A}=\int_{B_{0}} W(\mathbf{F}, \nabla \mathbf{F}) \mathrm{d} B_{0}=\int_{B_{0}}\left(W_{\mathrm{I}}(\mathbf{F})+W_{\text {II }}(\mathbf{F}, \nabla \mathbf{F})\right) \mathrm{d} B_{0},
$$

where $W_{\text {I }}$ and $W_{\text {II }}$ are constitutively given by (7) and (11), respectively. Assuming the previous expression for the action functional implies that all inertia effects are neglected and that we are hence considering a static case. As it will be shown in the remainder of the paper, this assumption is sensible for the applications which are targeted here.

\subsection{Second-gradient theory as the limit case of a micromorphic theory}

In this subsection, we will present the principle of virtual powers for the considered second-gradient material passing through the theory of micromorphic media. The theory of micromorphic media (see $[7,8])$ is known to be suitable to account for microstructure in elastic materials. This theory is more general than a second-gradient one in the sense that the set of unknown kinematical fields is enriched with respect to the classical kinematics based on the displacement field alone. More precisely, supplementary kinematical fields accounting for the motion of the microstructure are provided thus generalizing the classical kinematical framework of Cauchy and second-gradient continua. In this paper, we state the principle of virtual powers for 3D composite interlocks by means of a simple micromorphic model and we use suitable Lagrange multipliers to let the considered micromorphic model tend to the second-gradient model presented in the previous sections. The interest of introducing the principle of virtual powers by means of this approach is threefold: (i) The presentation via a micromorphic model allows to better catch the physical meaning of the considered internal and external actions, (ii) the natural and kinematical boundary conditions which can be used naturally take an intuitive meaning, and (iii) last but not least, the numerical implementation of the considered generalized problem is easier and the obtained solution is more stable. As far as considering the third quoted advantage of using constrained micromorphic theories to numerically implement second-gradient problems, one has to notice that the gain in terms of numerical calculations is evident. Indeed, when considering a differential problem stemming from a micromorphic model, the associated differential equations are of lower order with respect to those which would directly 
derive from a second-gradient model (second order vs. fourth order). These lower-order equations are obviously easier to be solved from a numerical point of view and the obtained numerical solution will be more stable and precise.

To proceed according to this optic, we introduce the kinematical fields of the considered micromorphic model by means of the two vector functions

$$
\chi: B_{0} \rightarrow \mathbb{R}^{3}, \quad \boldsymbol{\psi}: B_{0} \rightarrow \mathbb{R}^{2},
$$

the first one being the classical placement field introduced before also for the second-gradient kinematics and the second one accounting for microscopic motions in the considered continuum. The micromorphic model proposed here is simpler than the classical one proposed by Mindlin and Eringen $[7,8]$ since we only consider here two additional scalar functions instead of the nine which are introduced in the quoted models. We hence introduce a micromorphic strain energy density which take the following particular form and which is used to implement our numerical simulations:

$$
\tilde{W}_{\mathrm{II}}(\nabla \boldsymbol{\psi})=\frac{1}{2} A_{S}^{t 1}\left|\nabla \psi_{1}\right|^{2}+\frac{1}{2} A_{S}^{t 2}\left|\nabla \psi_{2}\right|^{2},
$$

where we denoted by $\psi_{\alpha}, \alpha=1,2$ the components of the vector $\boldsymbol{\psi}$. By direct comparison of the energies (12) and (11) it can be checked that the proposed micromorphic energy tends to the second-gradient one introduced before if $\psi_{1} \rightarrow i_{9}$ and $\psi_{2} \rightarrow i_{10}$. In order to account for such constraints in the weak formulation of the problem, we introduce suitable Lagrange multipliers $\Lambda_{1}$ and $\Lambda_{2}$ which have an associated energy density of the type

$$
W_{\mathrm{L}}(\mathbf{F}, \boldsymbol{\psi}, \boldsymbol{\Lambda})=\Lambda_{1}\left(\psi_{1}-i_{9}\right)+\Lambda_{2}\left(\psi_{2}-i_{10}\right),
$$

where we clearly set $\boldsymbol{\Lambda}=\left(\Lambda_{1}, \Lambda_{2}\right)$.

We hence propose to write the action functional of the proposed micromorphic medium as

$$
\mathcal{A}=\int_{B_{0}}\left(W_{\mathrm{I}}(\mathbf{F})+\tilde{W}_{\text {II }}(\nabla \boldsymbol{\psi})+W_{\mathrm{L}}(\mathbf{F}, \boldsymbol{\psi}, \boldsymbol{\Lambda})\right) \mathrm{d} B_{0},
$$

where $W_{\mathrm{I}}$ is the same energy given in $(7)$, while the energies $\tilde{W}_{\mathrm{II}}$ and $W_{\mathrm{L}}$ are introduced in terms of the additional kinematical variables as in formula (12) and (13), respectively. The power of internal forces of the considered constrained micromorphic medium can be written as the first variation of the considered action functional as

$$
\mathcal{P}^{\text {int }}=\delta \mathcal{A}=\int_{B_{0}}\left(\left(\frac{\partial W_{\mathrm{I}}}{\partial F_{i j}}+\frac{\partial W_{\mathrm{L}}}{\partial F_{i j}}\right) \delta F_{i j}+\frac{\partial W_{\mathrm{L}}}{\partial \psi_{\alpha}} \delta \psi_{\alpha}+\frac{\partial \tilde{W}_{\mathrm{II}}}{\partial \psi_{\alpha, j}} \delta \psi_{\alpha, j}+\frac{\partial W_{\mathrm{L}}}{\partial \Lambda_{\alpha}} \delta \Lambda_{\alpha}\right) .
$$

The power of external forces is easily introduced when considering a micromorphic framework (see, e.g., [9]) and in the present case, neglecting body external actions, can take the form

$$
\mathcal{P}^{\text {ext }}=\int_{\partial B_{0}}\left(f_{i}^{\text {ext }} \delta \chi_{i}+\tau_{\alpha} \delta \psi_{\alpha}\right) .
$$

Indeed, in the performed numerical simulations, we assume that the virtual fields $\delta \psi_{\alpha}$ are arbitrary on the boundary of the considered specimen (vanishing double force: $\tau_{\alpha}=0$ ), while the virtual displacement $\delta \chi_{i}$ is arbitrary almost everywhere, except on small subparts of the boundary where the displacement is assigned or vanishing. Such small parts of the boundary on which the displacement is vanishing can eventually change during deformation as happens for the contact of simply supported interlocks undergoing large bending deformations (for examples of modeling of contact laws see e.g., [54-56]). The boundary conditions to be applied to model contact between two deformable continua is of difficult implementation, but contact laws are usually already implemented in numerical codes as, e.g., COMSOL ${ }^{\circledR}$. We used such tool to model the contact in our numerical three-point bending simulations. 
The weak formulation of the differential problem for the considered constrained micromorphic medium can be then stated as

$$
\mathcal{P}^{\text {int }}=\mathcal{P}^{\text {ext }},
$$

where the internal and external power are, respectively, given by (14) and (15).

It is worth to remark that starting from this formulation of the principle of virtual powers and considering arbitrary variations $\delta \Lambda_{i}$ of the Lagrange multipliers, one gets the bulk constraints which actually let the considered micromorphic model tend to the particular second-gradient one previously introduced, namely

$$
\psi_{1}=i_{9}, \quad \psi_{2}=i_{10}
$$

It is clear that starting from the principle of virtual powers and integrating by parts, one could also obtain the strong form of the bulk equations and naturally associated boundary conditions in duality of the virtual variations $\delta \chi_{i}$ and $\delta \psi_{\alpha}$. Nevertheless, since the numerical simulations presented in the following are directly implemented via the weak form (16), we do not explicitly write here such strong equations.

\section{Numerical simulations for three-point bending of composite interlocks}

In this section, we present the numerical results stemming from the application of the proposed secondgradient model to the case of three-point bending of a composite interlock. The first-gradient constitutive parameters appearing in equation 8 are assumed to take the values presented in Tables 1, 2 and 3, in agreement with the experimental identification proposed in [35]. We remark that the two out-of-plane shear potentials are not symmetric in the sense that the corresponding constants appearing in Table 3 do not take the same values for the two orders of yarns. This fact is due to different weaving patterns in the warp and weft directions and has been experimentally observed in [35].

Indeed, it must be said that the constitutive expressions for the in-plane and out-of-plane shear potentials (last three equations in (8)) are slightly different from the ones used in [35]. Nevertheless, the associated stresses (derivatives of the energy with respect to $I_{\mathrm{sh}}^{p}, I_{\mathrm{sh}}^{t 1}$ and $I_{\mathrm{sh}}^{t 1}$ ) are seen to be almost equivalent. The reason to introduce here a simplified expression for the shear potentials is that a quadratic energy (linear stress) is more easily treated in numerical calculation than an energy in which non-integer

TABLE 1. Constitutive parameters appearing in the elongation and compression energy potentials

\begin{tabular}{llllll}
\hline$K_{\text {elong }}^{0}$ & $K_{\text {elong }}$ & $I_{\text {elong }}^{0}$ & $K_{\text {comp }}$ & $I_{\text {comp }}^{0}$ & $q$ \\
$37.85 \mathrm{MPa}$ & $816.33 \mathrm{MPa}$ & 0.0145 & $7.57 \times 10^{-3} \mathrm{MPa}$ & -1.12 & 2.85 \\
\hline
\end{tabular}

TABLE 2. In-plane shear constitutive parameters

\begin{tabular}{lllll}
\hline$K_{\text {shp }}^{12}$ & $K_{\text {shp }}^{21}$ & $p$ & $I_{\text {shp }}^{0}$ & $W_{\text {shp }}^{0}$ \\
$0.07575 \mathrm{MPa}$ & $1.69 \times 10^{-4} \mathrm{MPa}$ & 3.69 & $4.2 \times 10^{-3}$ & $-1.69 \times 10^{-4} \mathrm{MPa}$ \\
\hline
\end{tabular}

TABLE 3. Out-of-plane shear constitutive parameters

\begin{tabular}{llll}
\hline$K_{\text {sht } 1}^{12}$ & $K_{\text {sht } 1}^{22}$ & $K_{\text {sht1 }}^{21}$ & $I_{\text {sht } 1}^{0}$ \\
\hline $0.064945 \mathrm{MPa}$ & $0.00401131 \mathrm{MPa}$ & $0.00079691 \mathrm{MPa}$ & $1.4 \times 10^{-2}$ \\
\hline$K_{\text {sht } 2}^{12}$ & $K_{\text {sht } 2}^{22}$ & $K_{\text {sht } 2}^{21}$ & $I_{\text {sht } 2}^{0}$ \\
\hline $0.0330351 \mathrm{MPa}$ & $0.0042497 \mathrm{MPa}$ & $0.000736072 \mathrm{MPa}$ & $3 \times 10^{-2}$ \\
\hline
\end{tabular}




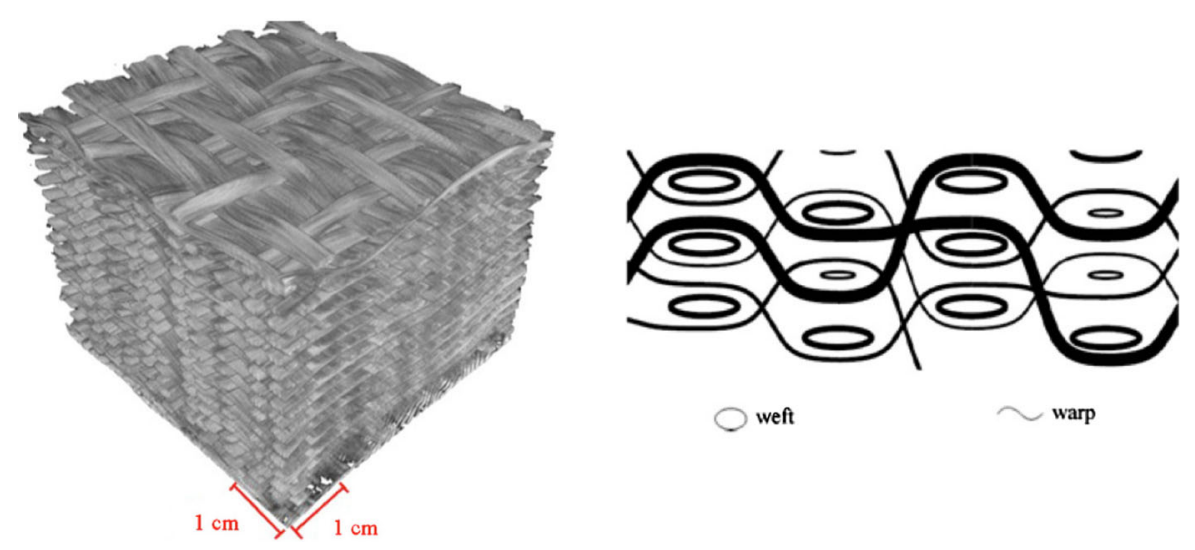

FIG. 2. Example of 3D woven composite interlock with an approximative weaving pattern

powers of the considered invariants appear. All other constitutive laws used in this paper (see Eq. (8)) coincide with the one proposed in [35].

The physical test we want to reproduce here is a simple three-point bending of a composite reinforcement beam with rectangular cross sections. The considered interlocks are 3D materials (see Fig. 2) in which a specific mesostructure with particular ordered patterns can be identified.

The basic architecture of an interlock fabric is shown in Fig. 2 (see also [57]). Two layers of weft yarns are joined by the weaving of the warp yarns. Consequently, all the yarns through the thickness are joined by the weaving. The resulting material is $3 \mathrm{D}$ with no third yarn set in the transverse direction but the properties through the thickness are much improved. In particular, problems related to the possible delaminations of the 2D laminated composites are overcome due to the described weaving procedure.

In this paper, we will focus on two different types of samples which basically differ one from the other for the direction of warp and weft with respect to the boundaries of the considered specimen. More particularly, we consider a three-point $0^{\circ} / 90^{\circ}$ bending test (warp and weft directions aligned with the edges of the specimen) and a three-point $\pm 45^{\circ}$ bending test (the yarn directions form an angle of $45^{\circ}$ degrees with respect to the longer sample edge). Numerical simulations showing the effect of the introduced second-gradient parameters will be proposed for both cases, and a discussion on the need of considering such a generalized continuum theory will be performed.

In all the performed numerical simulations, we consider specimens with the dimensions of $200 \times 30 \times$ $15 \mathrm{~mm}$, and we impose in the middle of the specimen a displacement of $60 \mathrm{~mm}$. As already discussed, since large deformations are imposed to the specimen, the contact law between the specimen and the two cylindrical supports is a crucial point for the correct modeling of the considered problem. More particularly, as far as boundary conditions are concerned, we suppose that external double forces are vanishing, while the force at the supports are assumed to follow a frictionless contact law which is builtin in the COMSOL ${ }^{\circledR}$ code. In the middle of the top surface, a displacement is applied which goes from 0 to $60 \mathrm{~mm}$.

As for the values of the second-gradient parameters appearing in Eq. (11), they are obtained by inverse approach, i.e., by fitting the performed second-gradient numerical simulations with the available experimental data concerning three-point bending of $0^{\circ} / 90^{\circ}$ and $\pm 45^{\circ}$ composite interlocks. Inverse approaches are commonly used in the calibration process of newly introduced constitutive parameters (see, e.g., [58-60]). As it will be seen in the following subsections, the values of the second-gradient parameters which are needed to fit at best the experimental evidences are not constant but depend on the entity of the macroscopic deformation. This indicates that the constitutive law (11) may not be general enough to catch all the possible material behaviors at high strains. Indeed, as far as sufficiently 

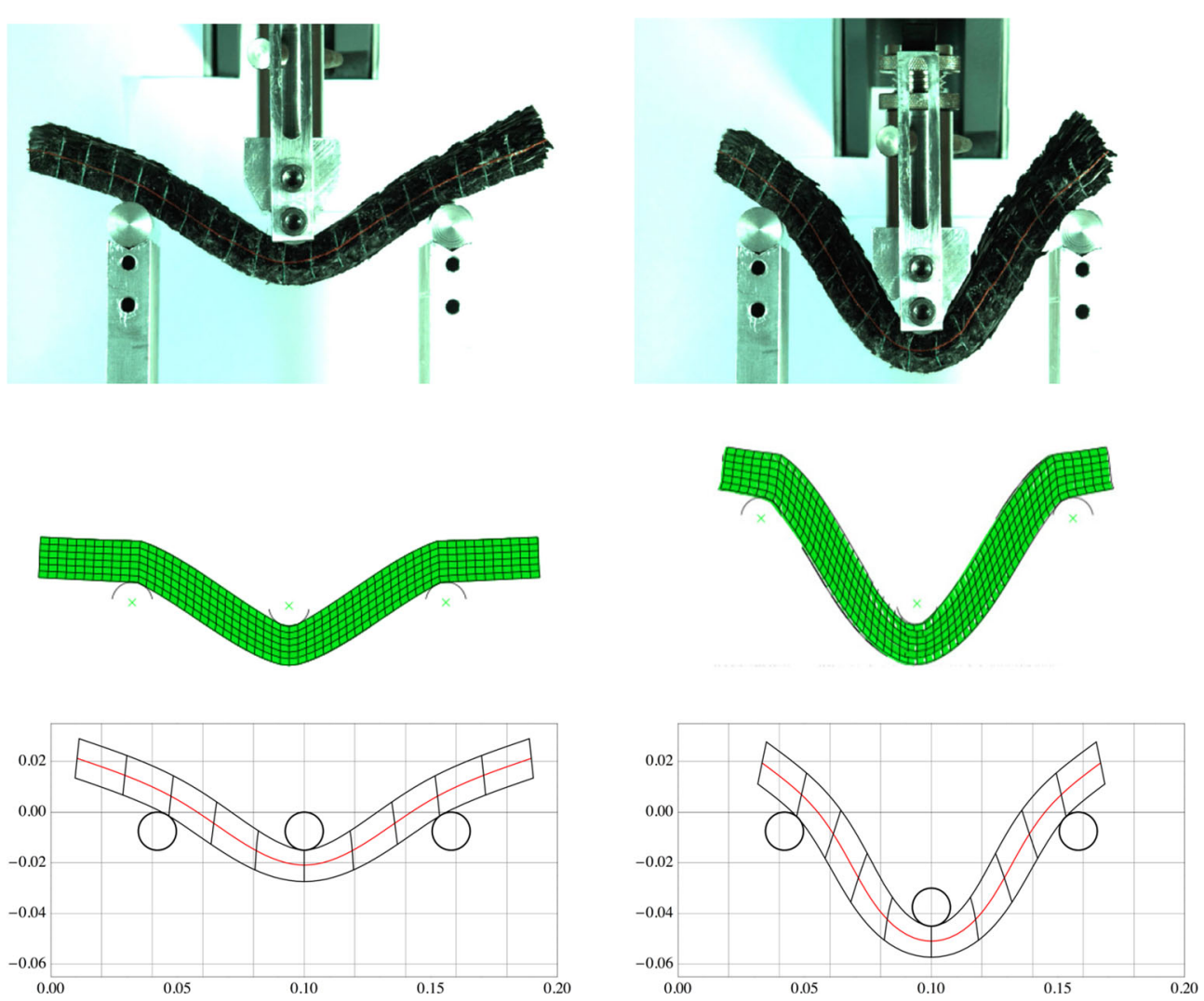

FIG. 3. $0^{\circ} / 90^{\circ}$ three-point bending for an imposed displacement of $30 \mathrm{~mm}$ (left) and of $60 \mathrm{~mm}$ (right). Top: experimental shapes, middle: first-gradient simulations, bottom: second-gradient simulations with $A_{S}^{t 1}=A_{S}^{t 2}=1.2 \times 10^{-5} \mathrm{MPa} \times \mathrm{m}^{2}($ left $)$ and $A_{S}^{t 1}=A_{S}^{t 2}=5 \times 10^{-6} \mathrm{MPa} \times \mathrm{m}^{2}$ (right). The scale for the $x$ and $y$ axes is $m$

small imposed displacements are considered (up to 30-40 mm), a quadratic constitutive law of the type (11) is sufficient to describe quite precisely the overall material behavior. On the other hand, when higher imposed displacements are considered, different values of the second-gradient parameters must be chosen to fit at best the experimental shape. Further studies on the formulation of the constitutive behavior of composite interlocks are thus needed which are focused on the development of more complex nonlinear second-gradient constitutive equations.

\subsection{Three-point $0^{\circ} / 90^{\circ}$ bending test: the effect of out-of-plane yarns' bending stiffness}

In this subsection, we present the numerical simulations obtained via the proposed linear second-gradient model and we compare the obtained solutions with those issued by the classical Cauchy theory. Figure 3 shows the comparison between the experimental tests [57], the first-gradient solution [61] and the secondgradient one. It can be immediately noticed that the first-gradient solution does not allow to correctly describe the deformation of the two ends of the beam whose deformation significantly deviates from the experimental data. This discrepancy between the experiments and the first-gradient solution can be better pointed out in Fig. 4. In this picture, the fact that the the two ends of the beam do not correctly lift up results to be quite evident. On the other hand, the better fitting of the second-gradient solution with the experimental evidence can be seen in Fig. 5. It can also be remarked in the quoted 


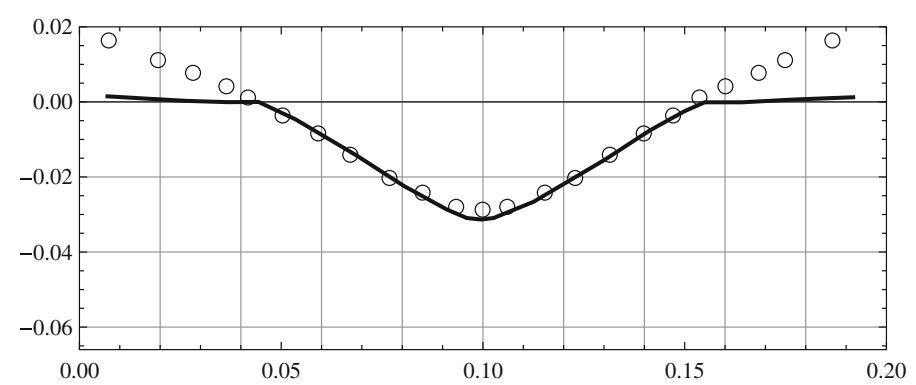

(a)

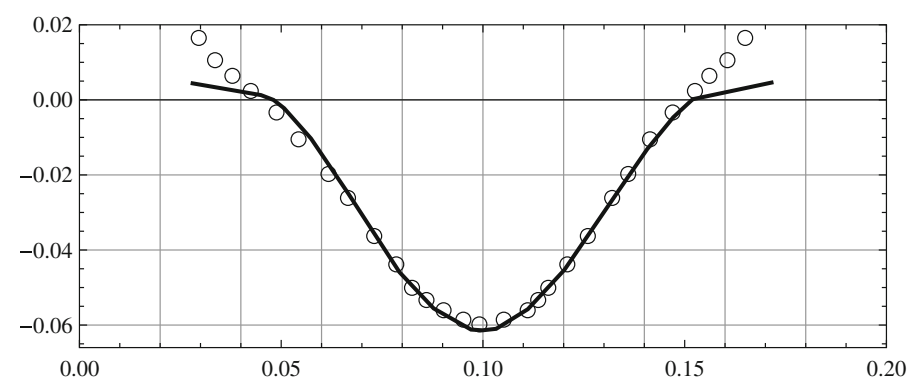

(b)

FIG. 4. Comparison between experimental data (dots) and first-gradient solution: current shape of the mean axis of the specimen for imposed displacement of $30 \mathrm{~mm}$ (a) and for $60 \mathrm{~mm}$ (b). The scale for the $x$ and $y$ axes is $m$

picture that the fitting between the second-gradient solution and the experimental data is performed by using two different values of the second-gradient parameter when considering small and high macroscopic deformations. These observations lead naturally to infer that the second-gradient out-of-plane parameters cannot be considered to be constant, but seem to vary with deformation. This allows to conjecture that the second-gradient constitutive law should indeed be generalized with respect to the simple quadratic form considered in Eq. (11) in order to include material nonlinearities also in the second-gradient terms. In this paper, we limit ourselves to the quadratic second-gradient constitutive law (11) which is able to catch the most important features of the mechanical behavior of considered interlocks with very few elastic constants. On the other hand, we also remark that in subsequent works, a generalized nonlinear constitutive law needs to be formulated for the second-gradient energy in order to complete the mechanical characterization of considered materials.

The results obtained by means of the performed numerical simulations are appealing as they strongly suggest that the presence of second-gradient terms in the strain energy density of the considered orthotropic material is unavoidable if one wants to correctly model the three-point bending of a $0^{\circ} / 90^{\circ}$ interlock while remaining in a continuum framework. Indeed, it is sensible that the out-of-plane bending stiffness plays a very important role in the deformation of such materials. In fact, the predominant deformation mode in such a test is related to the bending deformation of the family of yarns which are aligned with the longer side of the specimen. The family of yarns aligned with the depth of the specimen has very little influence on the global deformation of the considered sample. The fact that the longer yarns bend and that they posses a non-negligible out-of-plane bending stiffness allows the two ends of the beam to lift up. Such a deformation pattern is well recovered by the second-gradient numerical simulations, but not by the first-gradient ones (see Fig. 3). Moreover, it can be seen in the quoted figure that as far as the three-point bending of a $0^{\circ} / 90^{\circ}$ specimen is considered, the cross sections of the beam are 


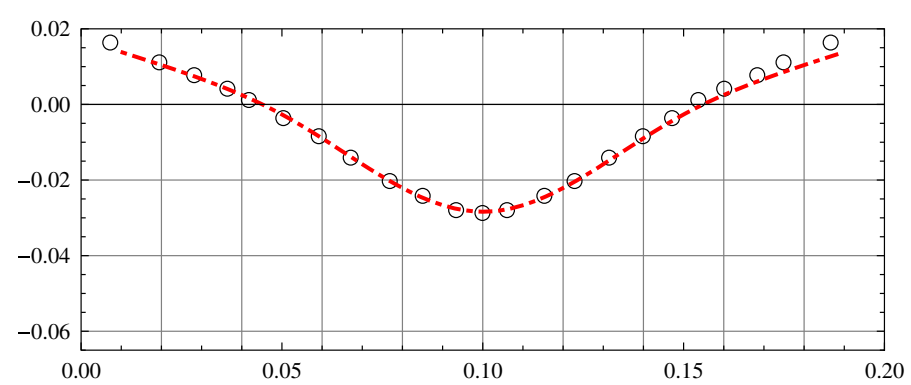

(a)

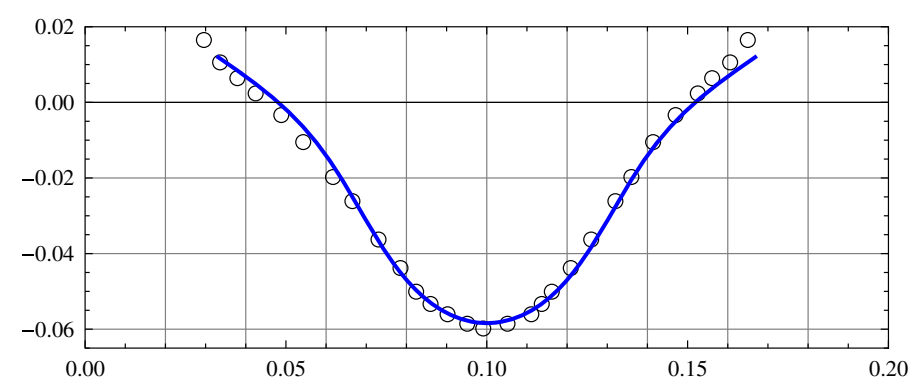

(b)

FIG. 5. Comparison between experimental data (dots) and second-gradient solution: current shape of the mean axis of the specimen for imposed displacement of $30 \mathrm{~mm}$ and $A_{S}^{t 1}=A_{S}^{t 2}=1.2 \times 10^{-5} \mathrm{MPa} \times \mathrm{m}^{2}(\mathbf{a})$ and of $60 \mathrm{~mm}$ and $A_{S}^{t 1}=A_{S}^{t 2}=$ $5 \times 10^{-6} \mathrm{MPa} \times \mathrm{m}^{2}(\mathbf{b})$. The scale for the $x$ and $y$ axes is $m$

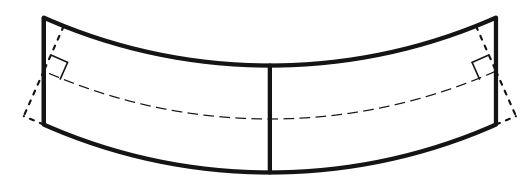

Fig. 6. Schematic representation of $0^{\circ} / 90^{\circ}$ bending test: Euler-Bernoulli hypothesis of cross sections orthogonal to the mean axis is violated

not orthogonal to its mean axis: This is directly related to the fact that the yarns can be considered almost inextensible in the considered woven composite. Indeed, as it can be seen in Fig. 6, a specimen which behave as an Euler-Bernoulli beam would need that the upper part of the specimen shrink and the lower part elongate in order to let the cross sections stay orthogonal with respect to the mean axis. This shrinking/elongation deformation of the specimen is not possible due to the quasi-inextensibility of the yarns: The inextensibility constraint actually imposes a relative sliding of the yarns and, as a result, a rotation of the cross sections with respect to the direction orthogonal to the mean axis.

\subsection{Three-point $\pm 45^{\circ}$ bending test}

The present subsection is devoted to the comparison between first- and second-gradient solutions for the $\pm 45^{\circ}$ three-point bending. Figure 7 shows a schematic representation of the bending of a $\pm 45^{\circ}$ specimen. It can be seen from this figure that in such a deformation pattern, the upper part of the specimen necessarily undergoes shrinking, while the bottom part is elongated instead. This change of 


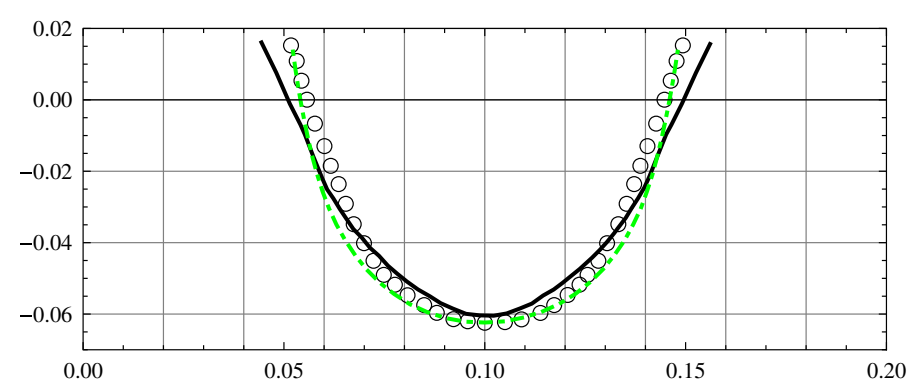

FIG. 9. Comparison of experimental data with different second-gradient solutions for an imposed displacement of $60 \mathrm{~mm}$ and $A_{S}^{t 1}=A_{S}^{t 2}=7.5 \times 10^{-6} \mathrm{MPa} \times \mathrm{m}^{2}$. The scale for the $x$ and $y$ axes is $m$

lift-up of the two ends of the beam takes place even in the case of a first-gradient theory is due to the fact that the fibers can rather easily rotate with respect to the vertical direction even without bending, due to their reduced length. Such almost rigid rotation of the fibers produces a non-local transmission of deformation which allows the two ends of the beam to partially lift-up. Nevertheless, a small amount of bending of the yarns can be seen to be present also in the $\pm 45^{\circ}$ case. Such effect of the shear bending stiffness can be recognized to be important both for the complete lift of the two ends of the beam and for the curvature of the middle part of the specimen.

Once again, we remark that the value of the second-gradient parameter used in the numerical simulations is not the same as the one used for the $0^{\circ} / 90^{\circ}$ test. This corroborates the thesis according to which the introduced simple constitutive load must be further generalized in order to account for nonlinear second-gradient material behaviors.

\section{Conclusions}

In this paper, we present an orthotropic 3D second-gradient model which is suitable to describe the complex mechanical behavior of thick composite interlocks. The need of using such generalized continuum theory is revealed by the study of the three-point bending of 3D fibrous composite reinforcements. The considered specimens are parallelepipeds with the dimensions of $200 \times 30 \times 15 \mathrm{~mm}$. Two types of specimens are considered which differ for the relative directions between the yarns and the edges of the specimen itself. More particularly, we consider the so-called $0^{\circ} / 90^{\circ}$ specimen in which one family of long yarns follows the direction of the longer edge of the specimen, while the second family of shorter yarns is directed along the depth of the specimen itself. The second type of specimen is called $\pm 45^{\circ}$ and is such that the yarns are directed at $\pm 45^{\circ}$ degrees with respect to the direction of the longer edge of the sample. In both cases, the specimens are subjected to a classical three-point bending test and the experimental results are compared with numerical solutions obtained via first- and second-gradient continuum theories. It appears clearly from the obtained results that the use of a second-gradient theory is a useful tool if one wants to correctly model the behavior of composite interlocks subjected to three-point bending. Indeed, we show that the fact of including the gradient of the out-of-plane shear angle variations in the strain energy density is directly related to the fact of considering the out-of-plane bending stiffness of the yarns at the mesoscopic level (and hence, indirectly, of the fibers at the microscopic level). Such bending rigidity of the mesostructure (microstructure) is crucial to correctly describe the response of the material to the applied external solicitations. In particular, we show that the out-of-plane bending of the yarns is one of the leading mesoscopic deformation mechanisms affecting the macroscopic bending of $0^{\circ} / 90^{\circ}$ specimens. In fact, when subjected to bending, such materials basically behave as a "package" of superimposed wires which are held together by a second family of shorter yarns which indeed do not intervene directly in the 
deformation process. The longer wires so bend all together (as happens for the pages of a book when one tries to bend it) and their bending rigidity allows to the two ends of the macroscopic specimen to lift up and to the middle part of the specimen to take its characteristic curvature. A first-gradient theory is not able to account for the bending of the mesostructure and hence is not suitable to correctly describe all the mechanisms which intervene in the complex process of deformation of composite interlocks. Moreover, we explicitly point out that the constraint of "quasi-inextensibility" of the yarns is directly related to the fact that a $0^{\circ} / 90^{\circ}$ specimen does not behave as an Euler-Bernoulli beam in the sense that cross sections, which are initially orthogonal to the mean axis of the beam, do not remain orthogonal during deformation. Indeed, due to large bending deformations, the upper and lower surface of the specimen inevitably have different curvature and cross sections could remain orthogonal to the mean axis if and only if a significant change of length occurred in the direction of the longer edge of the specimen. This is actually impossible, due to the fact that the yarns have a very high extensional rigidity.

Similar reasonings can be repeated also for the $\pm 45^{\circ}$ specimens, even if the deformation of such materials is more complex to be understood from a phenomenological point of view. We prove that in such a case, the bending rigidity of the yarns intervenes much less on the macroscopic deformation of the specimen, even if it keeps playing some role. Indeed, the main part of the out-of-plane motion of the yarns is accounted for by simple (quasi-rigid) rotations of the yarns with no (or very few) associated bending. Such a deformation mode is possible due to the fact that the yarns are relatively short with respect to the size of the specimen and they can rotate rather easily with respect to the vertical direction. Nevertheless, the fact of considering the bending of the yarns still bring some complements to the complete description of the experimental behavior of the considered materials, both for what concerns the complete lift of the two ends of the specimen and its global curvature. We finally remark that the $\pm 45^{\circ}$ specimens almost behave as Euler-Bernoulli beams. This is possible since changes of length of the specimen are allowed due to pantographic motions of the yarns.

We can summarize by saying that 3D composite interlocks can be correctly modeled accounting for the bending stiffness of the yarns by means of second-gradient theories. When considering three-point bending tests, the influence of the out-of-plane bending stiffness is much higher for $0^{\circ} / 90^{\circ}$ specimens than $\pm 45^{\circ}$ ones. We can infer that for orthotropic media with different initial angles between the yarns and the sample edges the effect of second-gradient terms would be of intermediate importance with respect to the two limit cases presented here.

The results proposed in this paper are a fundamental step for the characterization of the mechanical behavior of thick composite interlocks for the impact that such results can have on the modeling of forming processes of complete engineering components. Nevertheless, the conception of more complex nonlinear, second-gradient constitutive laws appears to be a crucial point for further investigations.

\section{References}

1. Piola, G.: The Complete Works of Gabrio Piola: Volume I Commented English Translation (Vol. 38). Springer, Berlin (2014)

2. Dell'Isola, F., Andreaus, U., Placidi, L.: At the origins and in the vanguard of peridynamics, non-local and highergradient continuum mechanics: an underestimated and still topical contribution of Gabrio Piola. Math. Mech. Solids (2014). doi:10.1177/1081286513509811

3. Mindlin, R.D., Eshel, N.N.: On first strain-gradient theories in linear elasticity. Int. J. Solids Struct. 4(1), 109-124 (1968)

4. Mindlin, R.D.: Second gradient of strain and surface-tension in linear elasticity. Int. J. Solids Struct. 1(4), 417-438 (1965)

5. Toupin, R.A.: Elastic materials with couple-stresses. Arch. Ration. Mech. Anal. 11(1), 385-414 (1962)

6. Toupin, R.A.: Theories of elasticity with couple-stress. Arch. Ration. Mech. Anal. 17(2), 85-112 (1964)

7. Mindlin, R.D.: Micro-structure in linear elasticity. Arch. Ration. Mech. Anal. 16(1), 51-78 (1964)

8. Eringen, A.C.: Microcontinuum Field Theories I: Foundations and Solids. Springer, Berlin (1999)

9. Bleustein, J.L.: A note on the boundary conditions of Toupin's strain-gradient theory. Int. J. Solids Struct. 3(6), 10531057 (1967) 
10. Neff, P., Ghiba, I.D., Madeo, A., Placidi, L., Rosi, G.: A unifying perspective: the relaxed linear micromorphic continuum. Contin. Mech. Thermodyn. 1-43 (2013)

11. Madeo, A., Neff, P., Ghiba, I.D., Placidi, L., Rosi, G.: Wave propagation in relaxed micromorphic continua: modeling metamaterials with frequency band-gaps. Contin. Mech. Thermodyn. 1-20 (2013)

12. Neff, P., Forest, S.: A geometrically exact micromorphic model for elastic metallic foams accounting for affine microstructure. Modelling, existence of minimizers, identification of moduli and computational results. J. Elast. 87(2-3), 239276 (2007)

13. Neff, P., Jeong, J., Münch, I., Ramézani, H.: Mean field modeling of isotropic random Cauchy elasticity versus microstretch elasticity. Zeitschrift für Angewandte Mathematik Und Physik 60(3), 479-497 (2009)

14. Neff, P., Jeong, J., Ramézani, H.: Subgrid interaction and micro-randomness-Novel invariance requirements in infinitesimal gradient elasticity. Int. J. Solids Struct. 46(25), 4261-4276 (2009)

15. Cosserat, E., Cosserat, F.: Théorie des Corps déformables. Paris (1909)

16. Germain, P.: La méthode des puissances virtuelles en mécanique des milieux continus-I: théorie du second gradient. J. Mécanique 12, 235-274 (1973)

17. Germain, P.: The method of virtual power in continuum mechanics. Part 2: microstructure. SIAM J. Appl. Math. 25(3), 556-575 (1973)

18. Ferretti, M., Madeo, A., dell'Isola, F., Boisse, P.: Modeling the onset of shear boundary layers in fibrous composite reinforcements by second-gradient theory. Zeitschrift für Angewandte Mathematik Und Physik 65, 587-612 (2013)

19. Forest, S.: Mechanics of generalized continua: construction by homogenizaton. Le J. de Physique IV 8, Pr8-39-48 (1998)

20. Kruch, S., Forest, S.: Computation of coarse grain structures using a homogeneous equivalent medium. Le J. de Physique IV 8, Pr8-197-205 (1998)

21. Forest, S.: Homogenization methods and mechanics of generalized continua-part 2. Theor. Appl. Mech. (28-29), 113-144 (2002)

22. Forest, S.: Homogenization methods and the mechanics of generalized continua. In: Maugin, G. (ed.) Geometry, Continua and Microstructure, Travaux en Cours No. 60, pp. 35-48. Hermann, Paris, France (1999)

23. Askes, H., Aifantis, E.C.: Gradient elasticity in statics and dynamics: an overview of formulations, length scale identification procedures, finite element implementations and new results. Int. J. Solids Struct. 48(13), 1962-1990 (2011)

24. Exadaktylos, G.E., Vardoulakis, I.: Microstructure in linear elasticity and scale effects: a reconsideration of basic rock mechanics and rock fracture mechanics. Tectonophysics 335(1), 81-109 (2001)

25. Eremeyev, V.A., Pietraszkiewicz, W.: Material symmetry group of the non-linear polar-elastic continuum. Int. J. Solids Struct. 49(14), 1993-2005 (2012)

26. Altenbach, H., Eremeyev, V.A., Lebedev, L.P., Rendon, L.A.: Acceleration waves and ellipticity in thermoelastic micropolar media. Arch. Appl. Mech. 80(3), 217-227 (2010)

27. Eremeyev, V. A., Lebedev, L.P., Altenbach, H.: Foundations of micropolar mechanics. Springer, Heidelberg (2013)

28. Piccardo G., Ranzi G., Luongo A.: A complete dynamic approach to the generalized beam theory cross-section analysis including extension and shear modes. Math. Mech. Solids (2013). doi:10.1177/1081286513493107

29. Yang, Y., Misra, A.: Micromechanics based second gradient continuum theory for shear band modeling in cohesive granular materials following damage elasticity. Int. J. Solids Struct. 49(18), 2500-2514 (2012)

30. Engheta, N., Ziolkowski, R.W.: Metamaterials: Physics and Engineering Explorations. Wiley, New York (2006)

31. Zouhdi, S., Sihvola, A.H., Vinogradov, A.P. (eds.): Metamaterials and Plasmonics: Fundamentals, Modelling, Applications. Springer, Berlin (2009)

32. Alibert, J.J., Seppecher, P., Dell'Isola, F.: Truss modular beams with deformation energy depending on higher displacement gradients. Math. Mech. Solids 8(1), 51-73 (2003)

33. Pideri, C., Seppecher, P.: A second gradient material resulting from the homogenization of an heterogeneous linear elastic medium. Contin. Mech. Thermodyn. 9(5), 241-257 (1997)

34. Dumont, J.P., Ladeveze, P., Poss, M., Remond, Y.: Damage mechanics for 3-D composites. Compos. Struct. 8(2), 119141 (1987)

35. Charmetant, A., Orliac, J.G., Vidal-Sallé, E., Boisse, P.: Hyperelastic model for large deformation analyses of 3D interlock composite preforms. Compos. Sci. Technol. 72(12), 1352-1360 (2012)

36. dell'Isola, F., Steigmann, D.: A two-dimensional gradient-elasticity theory for woven fabrics. J. Elast. 1-13 (2014)

37. Spencer, A.J.M., Soldatos, K.P.: Finite deformations of fibre-reinforced elastic solids with fibre bending stiffness. Int. J. Non-Linear Mech. 42(2), 355-368 (2007)

38. Luongo, A.: On the amplitude modulation and localization phenomena in interactive buckling problems. Int. J. Solids Struct. 27(15), 1943-1954 (1991)

39. Luongo, A.: Mode localization in dynamics and buckling of linear imperfect continuous structures. Nonlinear Dyn. 25(13), 133-156 (2001)

40. Luongo, A., Di Egidio, A.: Bifurcation equations through multiple-scales analysis for a continuous model of a planar beam. Nonlinear Dyn. 41(1-3), 171-190 (2005) 
41. Seppecher, P., Alibert, J.J., dell'Isola, F.: Linear elastic trusses leading to continua with exotic mechanical interactions. J. Phys.: Conf. Ser. 319:1, 012018 (2011)

42. Boutin, C., Hans, S.: Homogenisation of periodic discrete medium: application to dynamics of framed structures. Comput. Geotech. 30(4), 303-320 (2003)

43. Ghazavizadeh, A., Rutledge, G.C., Atai, A.A., Ahzi, S., Rémond, Y., Soltani, N.: Micromechanical characterization of the interphase layer in semi-crystalline polyethylene. J. Polym. Sci. Part B: Polym. Phys. 51(16), 1228-1243 (2013)

44. Michel, J.C., Moulinec, H., Suquet, P.: Effective properties of composite materials with periodic microstructure: a computational approach. Comput. Methods Appl. Mech. Eng. 172(1), 109-143 (1999)

45. Moulinec, H., Suquet, P.: Comparison of FFT-based methods for computing the response of composites with highly contrasted mechanical properties. Phys. B: Condens. Matter 338(1), 58-60 (2003)

46. Sanchez-Palencia, E.: Homogenization method for the study of composite media. In: Asymptotic Analysis II, pp. 192214. Springer, Berlin (1983)

47. Oshmyan, V.G., Patlazhan, S.A., Remond, Y.: Principles of structural-mechanical modeling of polymers and composites. Polym. Sci. Ser. A 48(9), 1004-1013 (2006)

48. Dagli, L., Remond, Y.: Identification of the non-linear behaviour a 4D carbon-carbon material designed for aeronautic application. Appl. Compos. Mater. 9(1), 1-15 (2002)

49. Mikdam, A., Makradi, A., Ahzi, S., Garmestani, H., Li, D.S., Remond, Y.: Statistical continuum theory for the effective conductivity of fiber filled polymer composites: effect of orientation distribution and aspect ratio. Compos. Sci. Technol. 70(3), 510-517 (2010)

50. Raoult, A.: Symmetry groups in nonlinear elasticity: an exercise in vintage mathematics. Commun. Pure Appl. Anal. 8(1), 435-456 (2009)

51. Schröder, J., Neff, P.: Invariant formulation of hyperelastic transverse isotropy based on polyconvex free energy functions. Int. J. Solids Struct. 40(2), 401-445 (2003)

52. Balzani, D., Neff, P., Schröder, J., Holzapfel, G.A.: A polyconvex framework for soft biological tissues. Adjustment to experimental data. Int. J. Solids Struct. 43(20), 6052-6070 (2006)

53. Schröder, J., Neff, P., Ebbing, V.: Anisotropic polyconvex energies on the basis of crystallographic motivated structural tensors. J. Mech. Phys. Solids 56(12), 3486-3506 (2008)

54. Andreaus, U., Chiaia, B., Placidi, L.: Soft-impact dynamics of deformable bodies. Continuum Mech. Thermodyn. 25(2-4), 375-398 (2013)

55. Cuomo, M., Ventura, G.: Complementary energy approach to contact problems based on consistent augmented Lagrangian formulation. Math. Comput. Model. 28(4-8), 185-204 (1998)

56. Turco, E.: A strategy to identify exciting forces acting on structures. Int. J. Numer. Methods Eng 64(11), 14831508 (2005)

57. Orliac, J.G.: Analyse et simulation du comportement anisotrope lors de la mise en forme de renforts tissés interlock. Ph.D. thesis, INSA-Lyon (2012)

58. Ghiba, I.D., Bulgariu, E.: On spatial evolution of the solution of a non-standard problem in the bending theory of elastic plates. IMA J. Appl. Math. (2013). doi:10.1093/imamat/hxt046

59. Ghiba, I.D.: Semi-inverse solution for Saint-Venant's problem in the theory of porous elastic materials. Eur. J. Mech.A/Solids 27(6), 1060-1074 (2008)

60. Bulgariu, E., Ghiba, I.D.: On the thermal stresses in anisotropic porous cylinders. Discret. Contin. Dyn. Syst. Ser. S 6, 1539-1550 (2013)

61. Charmetant, A.: Approches hyperélastiques pour la modélisation du comportement mécanique de préformes tissées de composites. Ph.D. thesis, INSA-Lyon (2011)

\author{
A. Madeo \\ LGCIE, INSA, Université de Lyon \\ 20 Avenue Albert Einstein \\ 69621 Villeurbanne Cedex \\ France \\ e-mail: angela.madeo@insa-lyon.fr
}

A. Madeo and F. dell'Isola

International Research Center M\&MoCS

Università dell'Aquila

L'Aquila, Italy 
M. Ferretti and P. Boisse

LaMCoS, INSA-CNRS, Université de Lyon

20 Avenue Albert Einstein

69621 Villeurbanne Cedex

France

e-mail: philippe.boisse@insa-lyon.fr

M. Ferretti

DICEAA, Università dell'Aquila

L'Aquila

Italy

e-mail: manuel.ferretti@insa-lyon.fr

F. dell'Isola

DISG, Università di Roma "La Sapienza"

Rome

Italy

e-mail: francesco.dellisola@uniroma1.it 